\title{
Estudo etnofarmacológico em entorno de floresta urbana como subsídio para a implantação da Fitoterapia no Sistema Único de Saúde
}

BORCARD, G.G. ${ }^{*}$; CONDE, B.E. ${ }^{1}$; ALVES, M.J.M.'; CHEDIER, L.M.'; PIMENTA, D.S.1.

${ }^{1}$ Universidade Federal de Juiz de Fora. Laboratório de Etnobotânica - Departamento de Botânica, Instituto de Ciências Biológicas. Rua José Lourenço Kelmer, S/n - Martelos, Juiz de Fora - MG, 36036-330 *Autor para correspondência: ggodinhomg@hotmail.com.

RESUMO: O estudo etnofarmacológico pode ser definido como exploração científica interdisciplinar dos agentes biologicamente ativos, tradicionalmente utilizados por populações humanas e que fazem parte de um acervo de conhecimento compartilhado. Desta forma o presente estudo teve como objetivo o estudo etnofarmacológico de plantas medicinais, no entorno de floresta urbana na Reserva Biológica Poço D'Anta em Juiz de Fora/MG visando a implantação da fitoterapia no Sistema Único de Saúde. Para este, realizou-se levantamento com três diferentes amostras: profissionais de saúde, domicílios em geral e especialistas locais. Quanto aos profissionais de saúde, pôde-se constatar que nenhum entrevistado soube conceituar o termo "Fitoterápico" e que não conheciam as políticas vigentes. Constatou-se que há aceitabilidade da implantação de Fitoterapia na saúde pública, porém, o conhecimento do tema é limitado. A partir das entrevistas nos domicílios em geral e com os especialistas locais, selecionou-se um total de 20 espécies botânicas para análise estatística e confirmação farmacológica. Esses resultados possibilitaram confrontar o conhecimento cultural com científico, com base em 14 espécies que poderiam ser cultivadas em horto na Reserva Biologica Poço D'Anta, com base em suas relevâncias locais. Os resultados obtidos podem subsidiar a aproximação do saber popular em relação ao científico, servindo de base para manutenção e fomento da implantação da Fitoterapia no sistema único de saúde.

Palavras-chave: etnofarmacologia, plantas medicinais e terapias complementares.

ABSTRACT: Ethnopharmacological study surrounding urban forest as a resource for the implementation of herbal medicine in the National Health System. The ethnopharmacological study can be defined as an interdisciplinary scientific exploration of biologically active agents, traditionally used by human populations and part of a shared body of knowledge. Thus, the current study focused on the ethnopharmacological research of medicinal plants, in the surroundings of the urban forest in the Biological Reserve PoçoD'Anta in Juiz de Fora / MG, aiming on the implementation of the herbal medicine in the Public Health System. For this purpose, a survey was held with three different groups: health professionals, members of the community and local experts. Concerning the health professionals, it could be verified that none of the participants were able to explain the term Phytotherapic and neither they had knowledge about the relevant and applicable policies. The acceptability for the implantation of Phytotherapy for public health use was observed, but the knowledge about this subject is limited. From the interviews with members of the community and local experts, a total of 20 plant species were selected for a statistical analysis and pharmacological confirmation. These results made possible to compare the cultural knowledge with the scientific one, defining 14 species that could be grown in the garden of the Biological Reserve Poço D'Anta, based on their local relevance. The results can support the approximation of the popular knowledge with the scientific one, providing a basis for the maintenance and promotion of the Phytotherapy in the Public Health System.

Keywords: ethnopharmacology, medicinal plants, complementary therapies. 


\section{INTRODUÇÃO}

Desde o início da civilização, o ser humano faz uso das plantas pela necessidade de sobrevivência, levando-o à descoberta de possíveis aplicações e potencialidades terapêuticas de determinadas espécies Lima et al. (2007).

No Brasil, a história da utilização de plantas medicinais (PM) não é diferente, pois as mesmas auxiliam no tratamento de doenças. Essa história apresenta influências da cultura indígena, europeia, africana e asiática, cujas marcas foram integradas num conjunto de princípios que visam à cura de doenças e restituem ao homem a vida natural Baldauf et al. (2009).

Como estratégia na investigação de $\mathrm{PM}$, tem-se a etnofarmacologia, que tem como objetivo resgatar e avaliar a eficácia das técnicas "tradicionais" sobre a utilização de plantas para fins terapêuticos, combinando informações adquiridas junto às comunidades que fazem uso da flora medicinal com estudos etnodirigidos, ou seja, com estudos etnobotânicos e etnofarmacológicos que visam contribuir na descoberta de novos farmácos (Albuquerque \& Hanazaki, 2006; Elisabetsky, 2003).

A saúde pública do Brasil foi aprimorada a partir do Sistema Único de Saúde (SUS) tendo como base a Atenção Primária à Saúde (APS), com diretrizes que criam uma forma de produzir as ações e serviços de saúde Gomes et al. (2011).

O presente estudo tem como objetivo avaliar o conhecimento sobre PM/ Fitoterapia da população residente ao entorno Reserva Biológica Poço D’Anta (ReBio Poço D'Anta), por parte dos usuários das unidades de saúde e profissionais de saúde que trabalham na Unidade de Atenção Primária a Saúde (UAPS), fornecendo subsídios para a implantação efetiva da PM/Fitoterapia no SUS.

\section{MATERIAIS E MÉTODOS \\ Caracterização da área}

ReBio Poço D'Anta é uma Unidade de Conservação de Proteção Integral, criada pelo Decreto Municipal 2.794, de 21 de setembro de 1982, com uma área de 277 ha, sendo considerada a primeira Unidade de Conservação criada no município de Juiz de Fora, MG, Brasil. As comunidades estudadas localizam-se ao entorno da ReBio e possuem Unidades de Atenção Primária à Saúde (UAPS) com Estratégia Saúde da Família (ESF).

Esse trabalho possui parecer favorável do Comitê de Ética em Pesquisa da Universidade Federal de Juiz de Fora, sob número 311/2009.

\section{Amostragem}

Foram escolhidos para as entrevistas: 1) profissionais da área de saúde que trabalham nas UAPS, tais como, médicos, enfermeiros, técnicos de enfermagem, dentistas, assistentes sociais e agentes comunitários de saúde (ACS); 2) moradores das comunidades (famílias cadastradas nas UAPS), com escolha a partir de sorteio aleatório simples, com base no programa Microsoft Excel ${ }^{\oplus}$, e que deu preferência ao morador mais velho (Conde, 2014a); e 3) especialistas locais (EL), que foram escolhidos com base nas técnicas "bola de neve" (Becker, 1993), onde os entrevistados foram escolhidos por indicação de outros entrevistados, tendo como ponto de partida o senhor responsável pelo horto municipal dentro da ReBio.

\section{Coleta de dados}

Foram realizadas entrevistas entre dezembro de 2011 e março de 2012, com aplicação de formulários para a coleta de dados etnofarmacológicos. Também se coletou material botânico, com posterior identificação pela equipe de pesquisadores do Departamento de Botânica da Universidade Federal de Juiz de Fora com posterior depósito de exsicatas no Herbário CESJ.

\section{Confirmação farmacológica}

Para realização da confirmação farmacológica e dados quantitativos, foram selecionadas as espécies que obtiveram citações igual ou maior que $6(+/-2 \%)$ e das quais foram obtidos espécimens férteis (Tabela 3 ).

As confirmações farmacológicas destas espécies foram confrontadas com literaturas científicas obtidas através de revisões realizadas no banco de dados Scopus.

\section{Análise estatística}

Para cada espécie foi calculada a Importância Relativa (IR) (Bennett \& Prance, 2000), que pode refletir a intensidade de uso de uma dada espécie, o que pode estar relacionado ao estado de conservação da mesma em uma dada área. Este cálculo tem como base a versatilidade, ou seja, uso da planta para mais de uma finalidade, sendo 2 o valor máximo obtido por uma espécie.

O cálculo foi feito de acordo com a fórmula $\mathrm{IR}=\mathrm{NSC}+\mathrm{NP}$, onde:

(NSC) $=n^{\circ}$ de sistemas corporais (categorias de doenças); NSC $=$ NSCE / NSCEV

NSCE = número de sistemas corporais tratados da espécie $\mathrm{x}$;

NSCEV = o número total de sistemas corporais tratados pela espécie mais versátil

$(\mathrm{NP})$ = número de propriedades

NP = NPE / NPEV;

NPE = número de propriedades atribuídas a espécie $\mathrm{x}$; 
NPEV = número total de propriedades atribuídas à espécie mais versátil.

Para isso, baseou-se cada uso terapêutico da espécie em uma categoria de doença na Classificação Estatística Internacional de Doenças e Problemas Relacionados à Saúde, $10^{\mathrm{a}}$ revisão (CID 10), e através da categoria buscou-se a propriedade correspondente (WHO, 1996).

Para se identificar os sistemas corporais que apresentaram maior importância nas entrevistas, foi utilizada a técnica adaptada de Trotter \& Logan (1986). Essa técnica é baseada no "consenso dos informantes".

O cálculo foi baseado na fórmula: $\mathrm{FCl}=$ nar - na/ nar - 1, onde:

$\mathrm{FCl}=$ fator de consenso dos informantes;

nar = somatório de usos registrados por cada informante para uma categoria;

na = número de espécies indicadas na categoria.

O valor máximo do $\mathrm{FCl}$ é 1, no qual ocorre um consenso completo entre os informantes a respeito de uma categoria medicinal para uma doença específica.

Os dados obtidos foram tabulados no programa Epi Info'⿳ versão 3.5.3 (Silva, 2013), e gerados gráficos pelo programa Microsoft Excel ${ }^{\circledR}$.

\section{RESULTADOS E DISCUSSÃO}

Foram entrevistados 142 profissionais de saúde, e se constatou que $91 \%$ dos profissionais eram do sexo feminino e a faixa de idade dos entrevistados foi de 40 a 59 anos (60\%). Estes perfil de entrevistados, também foi estudado por Conde et al. (2014 b) no mesmo município.

Ao serem questionados como definiam Fitoterapia, a resposta mais encontrada, com $31,2 \%$, foi "Plantas medicinais", seguida de "Medicamento natural" com 19,14\%. Pode-se considerar que nenhum dos entrevistados citou uma resposta de acordo com aAgência Nacional de Vigilância Sanitária (Brasil, 2006a), que considera como "medicamento fitoterápico aquele obtido exclusivamente de matérias-primas de origem vegetal, com qualidade constante e reprodutível e que tanto os riscos quanto à eficácia seja caracterizada por levantamentos etnofarmacológicos, documentações técnicocientíficas em publicações ou ensaios clínicos" (Brasil, 2006a). A ESF trabalha com educação continuada, e o fato destes profissionais estarem com essa conceituação deficitária aponta a necessidade de nivelamento teórico para iniciar-se um trabalho efetivo de Fitoterapia nos municípios onde os profissionais não apresentam conhecimento sobre este tipo de terapia.
A escolaridade dos profissionais de saúde foi avaliada em função da atuação. Entre os ACSs e os técnicos de enfermagem, predominou o ensino médio $(70 \%)$. Com relação aos profissionais com curso superior, tem-se que $86 \%$ se destacam já tendo feito alguma pós graduação.

Os profissionais de saúde também foram questionados acerca da origem de seus conhecimentos sobre PM/Fitoterapia. Verificou-se que apenas $13 \%$ dos entrevistados adquiriram seus conhecimentos nas universidades, entre médicos, enfermeiros e dentistas. A maioria dos profissionais, $58,4 \%$ dos 142 entrevistados, são autodidatas sobre o tema.

Os médicos percebem que os pacientes fazem uso de PM/Fitoterapia, porém, é limitado o interesse dessa classe em conhecer mais sobre PM/ Fitoterapia e, assim, poder aliá-los ao tratamento convencional, a fim de promover o melhor tratamento ao paciente. Desta forma, fica claro o distanciamento médico/paciente, comprometendo o objetivo da ESF, o que evidencia a necessidade de melhor formação em relação à PM/Fitoterapia por parte de prescritores.

Os resultados encontrados coincidem com o relatado por Rosa et al. (2011), no qual descrevem que a Fitoterapia ainda não faz parte do currículo do curso de saúde de maneira geral e que a introdução de cursos sobre terapias alternativas em faculdades de saúde é raríssima, embora o relatório da Comissão Interministerial de Planejamento e Coordenação do Governo Brasileiro (CIPLAN) de 1988, tenha recomendado a inclusão de conhecimento de práticas alternativas no currículo de ensino em saúde (Rosa et al., 2011). Os autores também citam que os profissionais se deparam com a Fitoterapia no exercício da profissão através da demanda dos próprios pacientes. As fontes informais de informação foram identificadas como predominantes, pois os profissionais buscam por informações que supram as necessidades com que se defrontam no cotidiano de trabalho na ESF.

Com relação ao conhecimento sobre as políticas relacionadas à Fitoterapia; especialmente a Política Nacional de Práticas Integrativas e Complementares (PNPIC) (Brasil, 2006a) e Política Estadual de Práticas Integrativas e Complementares (PEPIC) (Brasil, 2006a), os entrevistados fizeram uma autoavaliação de seus conhecimentos. Eles foram convidados a relacionar seus conhecimentos com notas de 0 , para nenhum conhecimento, a 10, com muito conhecimento. O percentual encontrado tanto para PNPIC e PEPIC ficou concentrado entre 0 a 3 com $69 \%$ e $80 \%$, respectivamente.

Quanto ao interesse em se aprender mais sobre a PNPIC, PEPIC e sobre o uso das PM/Fitoterapia, também classificando o nível de

Rev. Bras. PI. Med., Campinas, v.17, n.4, supl. II, p.928-936, 2015. 
interesse em 0 , quando não havia nenhum interesse, a 10 , como de muito interesse, observou-se que $80 \%$ dos entrevistados classificaram seu nível de interesse entre 8 e 10, o que demonstra resultado positivo às políticas. A principal justificativa para a falta de interesse em aprender mais sobre a PNPIC, PEPIC e sobre Fitoterapia foi falta de tempo dos profissionais para se dedicarem ao aprendizado e aperfeiçoamento. Verificou-se que o conhecimento dos profissionais entrevistados sobre PNPIC e PEPIC é básico. Pode-se, assim, concluir que há déficit na divulgação de políticas que visam fortalecer e contribuir com a Fitoterapia, fato esse preocupante, tendo em vista que as práticas complementares visam buscar seu lugar na área da saúde. De acordo com o trabalho realizado entre os profissionais de saúde do município de Anápolis, Goiás, a PNPIC, formalizada em 2006, é totalmente desconhecida entre os entrevistados, nenhum participante afirmou conhecê-la. Após explicação de seu teor, a mesma é amplamente aceita por todos os profissionais, o que demonstra que possivelmente esses programas ainda não estão difundidos como deveriam (Dutra, 2009). O estudo realizado por Sousa et al. (2012) corrobora o presente trabalho ao mostrar que a Fitoterapia no SUS ainda é limitada. Nos estados estudados através do Cadastro Nacional dos Estabelecimento de Saúde (CNES) e no Sistema de Informações Ambulatoriais (SIA), não foi possível encontrar profissionais cadastrados em Fitoterapia, somente nas áreas de acupuntura e homeopatia, dentro de práticas integrativas e complementares Sousa et al. (2012).

Apesar desse pouco conhecimento dos profissionais sobre PM/Fitoterapia vinculada ao SUS, na via relação com os usuários, $83 \%$ dos entrevistados admitiram conhecer usuários das UAPS que utilizam PM/Fitoterapia por conta própria. Além disso, $40 \%$ dos médicos e técnicos de enfermagem relataram prescrever ou indicar o uso de PM/Fitoterapia com a periodicidade de "algumas vezes". Isso é preocupante, visto que a maioria dos profissionais demonstrou não possuir conhecimento sobre PM/Fitoterapia, sendo assim, provavelmente desconhecem a dose adequada, a correta forma de preparo e as interações medicamentosas. $O$ intercâmbio entre conhecimento popular e ciência é urgente, uma vez que os usuários estão praticando a automedicação na medida em que utilizam PM/ Fitoterapia por conta própria, podendo ocorrer riscos à saúde do indivíduo, como o retardamento do conhecimento de algum distúrbio, dose inadequada ou excessiva, desconhecimento das possíveis interações medicamentosas, possibilidade de reações adversas e não efetividade do tratamento, dentre outras consequências (Carvalho, 2008). Por outro lado, podem ocorrer tratamentos com
$\mathrm{PM} /$ Fitoterapia mais efetivos que os convencionais (Brasil, 2012).

Os profissionais acreditam no uso da Fitoterapia, o que é um ponto relevante para consolidação desta prática no município estudado, além de incentivar esta prática em outros centros de saúde do Brasil. De acordo com Dutra (2009), a ampla aceitação pela maioria dos profissionais do uso de Fitoterápicos no SUS deve-se justamente ao baixo custo, determinado pelo fácil acesso, principalmente com relação às $\mathrm{PM}$, pois muitas delas podem ser encontradas ou cultivadas no próprio domicílio ou podem ser encontradas em feiras, mercados e barracas podendo ser uma alternativa a famílias de baixa renda com valorização do saber cultural local.

Em relação à realização de prescrição ou indicação de PM/Fitoterápicos, a opção "Nunca" foi mais citada entre os ACSs (43\%), enfermeiros (37\%) e dentistas $(75 \%)$, entre os médicos e os técnicos de enfermagem a opção "Algumas vezes" alcançou cerca de $40 \%$ das respostas.

Foram entrevistados 102 moradores cadastrados na UAPS, em que se constatou que $47 \%$ dos entrevistados que utilizam PM/Fitoterapia não informam ao médico este uso, o que sugere novamente o autotratamento. Automedicação foi observada em Boa Esperança-PR, no qual 41\% dos pacientes a realizam. Esse fato pode estar relacionado com a crença da população de que PM/ Fitoterapia, por serem produtos de origem natural, sejam seguros e não possuam efeitos colaterais Valeze et al. (2011).

Foram entrevistados 32 EL e constatou-se que $95 \%$ destes repassam seu conhecimento para outros, principalmente para vizinhos e familiares. De acordo com Oliveira et al. (2010), o conhecimento das propriedades terapêuticas das PM/Fitoterapia é adquirido através dos antepassados por transmissão oral, o que representa importante aspecto cultural. Além do que, é indispensável valorização e incentivo para a perpetuação do conhecimento destes EL, como sugerido por Conde et al. (2014 b), já que estes EL, são os maiores detentores do acervo de conhecimentos etnofarmacológicos nestes locais. Com base nos questionamentos realizados, pode-se propor como estratégia de implantação da Fitoterapia no SUS, a realização de cursos de nivelamento aos profissionais, os quais já demonstram aceitação, interesse e empatia com esta terapêutica.

Com relação ao perfil epidemiológico da área estudada, tem-se que as doenças mais citadas pelos entrevistados foram Hipertensão (Doença do aparelho Circulatório) e Diabetes (Doenças endócrinas, nutricionais e metabólicas), fato este confirmado pelo SIAB (Sistema de Informação de Atenção Básica) nas UAPS participantes do estudo,

Rev. Bras. PI. Med., Campinas, v.17, n.4, supl. II, p.928-936, 2015. 
no qual se tem que hipertensão é a doença que mais prevalece seguida de diabetes (Brasil, 2013), como podem ser vistos na Tabela 1.

\section{Confirmação Farmacológica e Dados Quantitativos das Análises das PM.}

Com a realização da confirmação da ação farmacológica comprovada frente ao conhecimento popular sobre o uso de PM/Fitoterapia, foi possível constatar que, na maioria das vezes, o uso popular coincide com estudos científicos confirmados por testes pré-clínicos, já que, das 20 espécies avaliadas, 14 foram confirmadas cientificamente quanto aos usos principais citados neste estudo (Tabela 2). As espécies não confirmadas são promissoras para estudos farmacológicos futuros com relação ao uso citado no presente trabalho.

Os resultados encontrados mostram a concordância da espécie Plantago major L. (IR 1,70) ao ser comparada com o estudo realizado por Vitalini et al. (2013), que, ao escolherem as 20 principais espécies mais citadas em seu estudo, apresentou para Plantago major (IR 1,61) a mesma finalidade.

Para se relacionar o presente trabalho com o da comunidade de Oeiras, estado do Piauí, é observado que a espécie Bidens pilosa L. apresenta as mesmas finalidades, icterícia e problemas renais, e valor semelhante de $I R=0,64$. O mesmo ocorre com a espécie Plectranthus barbatus Andrews, que apresenta, como uma de suas finalidades, desconforto abdominal e valor de $I R=0,57$, e com a espécie Ruta graveolens L., que apresenta como uma de suas finalidades ação anti-inflamatória e valor de IR=0,57. Esses resultados reafirmam que estas espécies têm, de fato, importância para a comunidade local na questão de sua finalidade, embora não apresentem alta versatilidade. A espécie Gossypium hirsutum L. (IR=0,79) também foi avaliada na comunidade de Oeiras, porém, com valor de IR inferior ao presente trabalho por apresentar menor versatilidade. A espécie Lippia alba (Mill.) N.E.Br. ex Britton \& P.Wilson $(\mathrm{IR}=1,14)$ apresentou, em ambos os estudos, alta versatilidade, tendo, como exemplo, a indicação para gripe e calmante Oliveira et al. (2010).

De acordo com o Fator de Consenso dos Informantes (FCl) (Tabela 3 ) foi verificado que o maior consenso de uso entre os informantes dentro da categoria medicinal foi a de algumas doenças infecciosas e parasitárias $(0,88)$, seguida por doenças do aparelho geniturinário $(0,85)$ e transtornos mentais e comportamentais $(0,83)$. Ao se relacionar estes resultados com a análise dos problemas de saúde (Tabela 2) foi observado que existe relação entre as citações das categorias, o que fortalece a importância de se disponibilizar espécies botânicas para estas finalidades, com destaque para as doenças Hipertensão e Diabetes. Estudo realizado por Vitalini et al. (2013), em Sondrio, Itália, apresenta os seguintes resultados para $\mathrm{FCl}$ : Doenças do aparelho respiratório $(0,88)$, doenças do aparelho digestivo $(0,86)$ e doenças do aparelho circulatório $(0,80)$, o que corrobora os resultados encontrados, uma vez que essas foram as principais citações de categorias de doenças. Resultado análogo foi referido no estudo realizado em Feira de Caruaru, Pernambuco, no qual se destacaram as categorias: doenças do

TABELA 1. Classificação das doenças encontradas entre os entrevistados e seus familiares de acordo com a CID - 10

\begin{tabular}{lcc}
\hline Categorias CID-10 & $\begin{array}{c}\text { \% de citações } \\
(\mathbf{n = 2 2 3 )}\end{array}$ & $\begin{array}{c}\text { Doença mais citada dentro da categoria (\% } \\
\text { citações da doença mais citada) }\end{array}$ \\
\hline Doenças do aparelho circulatório & 43,05 & Hipertensão $(94,79)$ \\
Doenças do aparelho respiratório & 7,63 & Bronquite $(64,70)$ \\
Doenças endócrinas, nutricionais e metabólicas & 27,80 & Diabetes $(75,80)$ \\
Doenças do aparelho digestivo & 1,34 & Gastrite $(66,66)$ \\
Doenças do aparelho geniturinário & 2,24 & Rins / Cálculo Renal $(40)$ \\
Doenças infecciosas e parasitárias & 0,45 & Hepatite $(100)$ \\
Doença do sist osteomuscular e conjuntivo & 8,52 & Artrose $(26,31)$ \\
Transtornos mentais e comportamentas & 3,14 & Depressão / Mental $(42,85)$ \\
Neoplasias & 1,79 & Câncer $(75)$ \\
Sintomas, anormais de exames clínicos & 0,45 & Dislexia $(100)$ \\
Doenças do Sistema nervoso & 2,69 & Alzheimer $(50)$ \\
Lesões, envenenamento & 0,45 & Alergia $(100)$ \\
Doenças do olho e anexos & 0,45 & Glaucoma $(100)$ \\
\hline
\end{tabular}

Legenda: Respostas dos entrevistados com relação às doenças encontradas entre os entrevistados e seus familiares, sendo estas classificadas de acordo com a CID - 10. 
TABELA 2. Espécies selecionadas para o estudo de revisão farmacológica em literatura científica e demais informações, a partir do levantamento etnofarmacológico

\begin{tabular}{|c|c|c|c|c|c|}
\hline $\begin{array}{l}\text { Nome científico/ popular } \\
\text { (número de citações) }\end{array}$ & $\begin{array}{l}\text { Uso principal (número de } \\
\text { citações) }\end{array}$ & $\begin{array}{l}\text { Parte } \\
\text { utilizada }\end{array}$ & $\begin{array}{l}\text { Forma de preparo } \\
\text { mais comum/ } \\
\text { administração }\end{array}$ & $\begin{array}{l}\text { Importância } \\
\text { Relativa (IR) }\end{array}$ & $\begin{array}{l}\text { Confirmação farmacológica } \\
\text { do uso relacionado ao uso } \\
\text { principal }\end{array}$ \\
\hline $\begin{array}{l}\text { Rosmarinus officinalis L./ } \\
\text { alecrim. Ringbom et al. (1998) }\end{array}$ & Calmante. Islam et al. (2005) & Folha & $\begin{array}{l}\text { Infusão/ oral e } \\
\text { tópico }\end{array}$ & 2,00 & $\begin{array}{l}\text { Antidepressivo. Sasaki et al. } \\
\text { (2013), Machado et al. (2009) }\end{array}$ \\
\hline $\begin{array}{l}\text { Leonurus sibiricus L./ Macaé. } \\
\text { Hennebelle et al. (2008) }\end{array}$ & $\begin{array}{l}\text { Disenteria. Ahmed et al. (2006), } \\
\text { e dores abdominais. Túrel et al. } \\
\text { (2009), }\end{array}$ & Folha & Maceração/ oral & 1,80 & $\begin{array}{l}\text { Antimicrobiano. Ahmed et } \\
\text { al. (2006); Analgésico e Anti } \\
\text { inflamatório. Islam et al. (2005) }\end{array}$ \\
\hline $\begin{array}{l}\text { Plantago major L./ transagem. } \\
\text { Goulart et al. (2007) }\end{array}$ & $\begin{array}{l}\text { Infecções gerais. Zétola et al. } \\
(2002)\end{array}$ & Folha & \multicolumn{2}{|c|}{$\begin{array}{l}\text { Infusão e decocção/ 1,70 } \\
\text { oral e tópico }\end{array}$} & $\begin{array}{l}\text { Antimicrobiano. Hetland et al. } \\
\text { (2000), Samuelsen (2000), } \\
\text { Velasco-Lezama et al. (2006); } \\
\text { Hepatoprotetor e Antiinflamatório. } \\
\text { Túrel et al. (2009), Ringbom et al. } \\
\text { (1998) }\end{array}$ \\
\hline $\begin{array}{l}\text { Lippia alba (Mill.) N.E.Br. ex } \\
\text { Britton \& P.Wilson / Melissa. } \\
\text { Silva et al. (2010) }\end{array}$ & $\begin{array}{l}\text { Calmante. Ringbom et al. (1998) } \\
\text { e gripe. Hetland et al. (2000) }\end{array}$ & Folha & Infusão/ oral & 1,46 & $\begin{array}{l}\text { Sedativo. Hennebelle et al. } \\
\text { (2008), Zétola et al. (2002) }\end{array}$ \\
\hline $\begin{array}{l}\text { Foeniculum vulgare Mill./ } \\
\text { funcho. Silva et al. (2010) }\end{array}$ & Calmante. Zétola et al. (2002) & Folha & $\begin{array}{l}\text { Infusão e decocção/ } \\
\text { oral }\end{array}$ & 1,36 & Não encontrado \\
\hline $\begin{array}{l}\text { Piper umbellatum L./ } \\
\text { pariparoba. Samuelsen (2000) }\end{array}$ & $\begin{array}{l}\text { Problemas renais. Ahmed et al. } \\
(2006)\end{array}$ & Folha & Decocção/ oral & 1,33 & $\begin{array}{l}\text { Afecções renais. Roersch (2010); } \\
\text { Problemas cardíacos, hepáticos e } \\
\text { renais. Agbor et al. (2012) }\end{array}$ \\
\hline $\begin{array}{l}\text { Gossypium hirsutum L./ } \\
\text { algodão. Roersch (2010) }\end{array}$ & $\begin{array}{l}\text { Infecções. Ahmed et al. (2006) } \\
\text { e problemas no ap. reprodutor } \\
\text { feminino. Túrel et al. (2009) }\end{array}$ & Folha & \multicolumn{2}{|c|}{$\begin{array}{l}\text { Infusão e decocção/ 1,16 } \\
\text { oral }\end{array}$} & Não encontrado \\
\hline $\begin{array}{l}\text { Rosa alba L./ rosa branca } \\
\text { Túrel et al. (2009) }\end{array}$ & $\begin{array}{l}\text { Problemas no ap. reprodutor } \\
\text { feminino. Velasco-Lezama et al. } \\
(2006) \text {, }\end{array}$ & Flor & Infusão/ oral & 1,10 & Não encontrado \\
\hline $\begin{array}{l}\text { Solidago chilensis Meyen/ } \\
\text { arnica. Goulart et al. (2007) }\end{array}$ & $\begin{array}{l}\text { Dores causadas por pancadas. } \\
\text { Túrel et al. (2009) }\end{array}$ & Folha & Infusão/ tópico & 1,10 & $\begin{array}{l}\text { Lombalgia. Silva et al. (2010); } \\
\text { Antiinflamatório. Goulart et al. } \\
\text { (2007), Liz et al. (2008), }\end{array}$ \\
\hline $\begin{array}{l}\text { Stachytarpheta cayennensis } \\
\text { (Rich.) Vahl / gervão } \\
\text { Samuelsen (2000) }\end{array}$ & $\begin{array}{l}\text { Depurativo do sangue. Ahmed et } \\
\text { al. (2006) }\end{array}$ & Folha & Infusão/ oral & 1,10 & $\begin{array}{l}\text { Hipocolesterolêmico. Chigozie } \\
\text { et al. (2008); hipoglicemiante. } \\
\text { Adebajo et al. (2007), Eliakim- } \\
\text { lkechukwu et al. (2010), }\end{array}$ \\
\hline $\begin{array}{l}\text { Bidens pilosa L./ picão. Túrel et } \\
\text { al. (2009) }\end{array}$ & $\begin{array}{l}\text { tlcterícia. Machado et al. (2009), } \\
\text { problemas renais. Hetland et al. } \\
(2000)\end{array}$ & Folha & Infusão/ oral & 0,90 & $\begin{array}{l}\text { Protetor do fígado. Yuan et al. } \\
(2008)\end{array}$ \\
\hline $\begin{array}{l}\text { Ocimum basilicum L./ alfavaca } \\
\text { Velasco-Lezama et al. (2006), }\end{array}$ & Gripe e tosse. Islam et al. (2005) & Folha & Decocção/ oral & 0,90 & Antiviral. Chiang et al. (2005) \\
\hline $\begin{array}{l}\text { Ruta graveolens L./ arruda } \\
\text { Velasco-Lezama et al. (2006), }\end{array}$ & $\begin{array}{l}\text { Antiinflamatório. Ahmed et al. } \\
(2006)\end{array}$ & Folha & $\begin{array}{l}\text { Infusão/ oral e } \\
\text { tópico }\end{array}$ & 0,90 & $\begin{array}{l}\text { Antiinflamatório. Rateesh et al. } \\
\text { (2013), Raghav et al. (2006), }\end{array}$ \\
\hline $\begin{array}{l}\text { Ocimum selloi Benth / erva } \\
\text { doce. Velasco-Lezama et al. } \\
(2006) \text {, }\end{array}$ & Calmante. Hetland et al. (2000) & Folha & Infusão/ oral & 0,80 & Não encontrado \\
\hline $\begin{array}{l}\text { Petroselinum crispum Mill.Fuss } \\
\text { / salsa. Samuelsen (2000) }\end{array}$ & $\begin{array}{l}\text { Cicatrizante. Ahmed et al. (2006) } \\
\text { e problemas no ap. reprodutor } \\
\text { feminino. Machado et al. (2009) }\end{array}$ & Raiz & Decocção/ oral & 0,80 & Não encontrado \\
\hline $\begin{array}{l}\text { Plectranthus barbatus Andrews } \\
\text { / boldo. Liz et al. (2008) }\end{array}$ & $\begin{array}{l}\text { Problemas hepáticos. Velasco- } \\
\text { Lezama et al. (2006) e } \\
\text { desconforto abdominal. Ringbom } \\
\text { et al. (1998) }\end{array}$ & Folha & Maceração/ oral & 0,63 & $\begin{array}{l}\text { Antioxidante e protetor hepático. } \\
\text { Maioli et al. (2010) }\end{array}$ \\
\hline $\begin{array}{l}\text { Persicaria hydropiper (L.) } \\
\text { Delarbre /erva de bicho. } \\
\text { Samuelsen, 2000) }\end{array}$ & $\begin{array}{l}\text { Hemorróida. Hetland et al. (2000) } \\
\text { e acaríase. Ahmed et al. (2006) }\end{array}$ & $\begin{array}{l}\text { Toda a } \\
\text { planta }\end{array}$ & Decocção/ tópico & 0,53 & Não encontrado \\
\hline $\begin{array}{l}\text { Costus spicatus (Jacq.)Sw.I } \\
\text { cana de macaco. Liz et al. } \\
(2008)\end{array}$ & $\begin{array}{l}\text { Problemas renais. Liz et al. } \\
(2008)\end{array}$ & Folha & $\begin{array}{l}\text { Infusão e decocção/ } \\
\text { oral }\end{array}$ & 0,46 & $\begin{array}{l}\text { Antiinflamatório. Quintans Junior } \\
\text { et al. (2010), Silva \& Parente } \\
(2003)\end{array}$ \\
\hline $\begin{array}{l}\text { Phyllanthus tenellus Roxb./ } \\
\text { rebenta pedra. Chigozie et al. } \\
(2008)\end{array}$ & $\begin{array}{l}\text { Problemas renais (Liz et al. } \\
(2008) \text {, }\end{array}$ & Folha & $\begin{array}{l}\text { Infusão e decocção/ } \\
\text { oral }\end{array}$ & 0,46 & $\begin{array}{l}\text { Antimicrobiano. Ignácio et al. } \\
(2001)\end{array}$ \\
\hline $\begin{array}{l}\text { Dysphania ambrosioides (L.) } \\
\text { Mosyakin \& Clemants/ erva de } \\
\text { santa Maria. Velasco-Lezama } \\
\text { et al. (2006), }\end{array}$ & $\begin{array}{l}\text { Antiparasitário (verme, piolho, } \\
\text { sarna e pulga). Velasco-Lezama } \\
\text { et al. (2006) }\end{array}$ & Folha & $\begin{array}{l}\text { Infusão/ oral e } \\
\text { passar na casa }\end{array}$ & 0,36 & $\begin{array}{l}\text { Antiparasitário. Kamel et al. } \\
(2011)\end{array}$ \\
\hline
\end{tabular}


TABELA 3. Fator de consenso dos informantes $(\mathrm{FCl})$ sobre as espécies utilizadas para análise estatística e confirmação farmacológica

\begin{tabular}{|c|c|c|c|}
\hline Categoria da doença conforme (CID 10) & $\begin{array}{c}\text { N. de } \\
\text { espécies }\end{array}$ & $\begin{array}{l}\text { N. de } \\
\text { citações }\end{array}$ & $\mathbf{F C l}$ \\
\hline Algumas doenças infecciosas e parasitárias & 2 & 10 & 0,88 \\
\hline Doenças do aparelho geniturinário & 10 & 63 & 0,85 \\
\hline Transtornos mentais e comportamentais & 6 & 30 & 0,83 \\
\hline $\begin{array}{l}\text { Sintomas, sinais e achados anormais de exames clínicos e de laboratório, não } \\
\text { classificados em outra parte }\end{array}$ & 6 & 24 & 0,78 \\
\hline Doenças do aparelho respiratório & 9 & 26 & 0,70 \\
\hline Doenças endócrinas, nutricionais e metabólicas & 2 & 3 & 0,50 \\
\hline Doenças do olho e anexos & 2 & 3 & 0,50 \\
\hline Doenças do aparelho circulatório & 7 & 12 & 0,45 \\
\hline Doenças do sangue e dos órgãos hematopoiéticos e alguns transtornos imunitários & 4 & 6 & 0,40 \\
\hline Doenças do aparelho digestivo & 5 & 7 & 0,33 \\
\hline Doenças do ouvido e da apófise mastóide & 1 & 1 & 0,00 \\
\hline Doenças da pele e do tecido subcutâneo & 2 & 2 & 0,00 \\
\hline Doenças do sistema osteomuscular e do tecido conjuntivo & 3 & 3 & 0,00 \\
\hline
\end{tabular}

Legenda: Fator de consenso dos informantes (FCl) sobre as espécies utilizadas para análise estatística e confirmação farmacológica.

aparelho respiratório $(0,68)$, transtornos mentais e comportamentais $(0,60)$ e doenças do aparelho geniturinário $(0,61)$ (Almeida \& Albuquerque, 2002).

\section{CONCLUSÃO}

Foi possivel concluir que os profissionais de saúde que trabalham nas UAPS possuem pouco conhecimento sobre PM/Fitoterapia e suas políticas, sendo relatado por eles o autodidatismo como meio mais comum de obtenção deste tipo de conhecimento. Contatou-se também, que a Fitoterapia não é oferecida à população do município de Juiz de Fora, MG, não havendo nenhum tipo de investimentos na capacitação de profissionais ou na construção de uma estrutura competentes em UAPS, demonstrando significativo distanciamento entre teoria e prática, pois desde 2006 a Fitoterapia está institucionalizada no SUS, porém ainda não implementada em UAPS.

No que se refere aos EL, fica evidente a importância de seus conhecimentos e da perpetuação destes para posteriores gerações. Com relação às $\mathrm{PM}$ mais citadas, verificou-se a importância quanto às categorias de doenças citadas no estudo. Desta forma, espera-se que os resultados dessa pesquisa possam subsidiar a implantação da Fitoterapia no SUS, através de cursos que enfatizem tal prática, incentivando os EL a divulgarem seus conhecimentos entre suas comunidades.

Considerando que a ESF tem como compromisso desenvolver suas ações de prevenção primordial da saúde junto à comunidade em geral e prevenção primária voltadas para clientela sadia, faz-se necessário a implementação dessas ações junto à comunidades como a do presente estudo.

\section{AGRADECIMENTOS}

Programa de Pós Graduação em Ciências Farmacêuticas, Programa Extensão Universitária "Produção de Plantas Medicinais e Fitoterapia PROEX-UFJF; Coordenação de Aperfeiçoamento de Pessoal de Nível Superior CAPES; Secretaria de Saúde - Prefeitura de Juiz de Fora

\section{REFERÊNCIAS}

ADEBAJO, A.C. et al. Hypoglycaemic constituents of Stachytarpheta cayennensis leaf. Planta médica. v. 73, n. 3, p.241-250, 2007.

AGBOR, G.A. et al. Piper species protect cardiac, hepatic and renal antioxidant status of atherogenic diet fed hamsters. Food Chemistry. v. 134, p. 1354-1359, 2012.

AHMED, F.; et al. Antibacterial activity of Leonurus sibiricus aerial parts. Phytotherapy. v. 77, n.4, p. 316-317, 2006.

ALBUQUERQUE, U.P.; HANAZAKI, N. As pesquisas etnodirigidas na descoberta de novos fármacos de interesse médico e farmacêutico: fragilidades e pespectivas. Revista Brasileira de Farmacognosia. n.16, p. 678-689, 2006.

ALMEIDA, C.F.C.B.R.; ALBUQUERQUE, U.P. Uso e conservação de plantas e animais medicinais no estado de Pernambuco (Nordeste do Brasil): um estudo de caso. Interciência. v. 27, n. 6, 2002.

BALDAUF, C. et al. "Ferveu, queimou o ser da erva": conhecimentos de especialistas locais sobre plantas

Rev. Bras. PI. Med., Campinas, v.17, n.4, supl. II, p.928-936, 2015. 
medicinais na região Sul do Brasil. Revista Brasileira de Plantas Medicinais. v.11, n.3, p.282-329, 2009.

BECKER, H.S. Métodos de pesquisa em ciências sociais. 1ed. HUCTEC. 1993. 178p.

BENNETT, B.C., PRANCE, G.T. Introduced plants in the indigenous pharmacopoeia of Northern South America. Economic Botany. v.54, n. 1, p.90-102, 2000.

BRASIL. Ministério da Saúde. Secretaria de Ciência, Tecnologia e Insumos Estratégicos. Departamento de Assistência Farmacêutica. A Fitoterapia no SUS e o Programa de Pesquisas de Plantas Medicinais da Central de Medicamentos. Brasília: Ministério da Saúde. p. 7. 2006a.

BRASIL. Ministério da Saúde. Portaria n 971, de 03 de maio de 2006. Aprova a Política Nacional de Práticas Integrativas e Complementares (PNPIC) no Sistema Único de Saúde. Lex: Diário Oficial da República Federativa do Brasil, Brasília, DF, seção 1, n. 84, 04 de maio de 2006b.

BRASIL. Ministério da Saúde. Secretaria de Atenção à Saúde. Departamento de Atenção Básica. Práticas Integrativas e Complementares. Plantas Medicinais e Fitoterapia na Atenção Básica. série A. Normas e Manuais Técnicos - Caderno de Atenção Básica, Brasília (DF): Ed. Ministério da Saúde.2012

BRASIL. Secretaria de Saúde. Sistema de Informação de Atenção Básica. Subsecretaria de Atenção Primária á Saúde. 7p. 2013.

CARVALHO, A.C.B. Situação do registro de medicamentos fitoterápicos no Brasil. Revista Brasileira de Farmacognosia, v. 18, n. 2, p. 314-319, 2008.

CHIANG, L.C.; NG, L.K.; CHENG, P.W.; CHIANG, W.; LIN, C.C. Antiviral activities of extracts and selected pure constituents of Ocimum basilicum. Clinical and Experimental Pharmacology and Physiolog., v. 32, n. 10, p.811-816, 2005.

CHIGOZIE, I,J., et al. The hypocholesterolemic effect of Stachytarpheta Cayennensis. Tea: Implications for the management of obesity and hypertension. Asian Journal of Biochemistry. v. 3, n.4, p. 267-270, 2008.

CONDE, B.E. et al. Synergy in ethnopharmacological data collection methods employed for communities adjacent to urban forest. Brazilian Journal of Pharmacognosy. v. 24, p. 425-432, 2014a.

CONDE, B.E.; et al. Ethnopharmacology in the vicinity of the Botanical Garden of the Federal University of Juiz de Fora, Brazil. Ethnobotany Research and Applications. 12: 91-111, $2014 \mathrm{~b}$.

DUTRA, M. G. Plantas medicinais e saúde pública: Um diagnóstico situacional em Anápolis, Goiás. 2009. 112p. Dissertação (Mestrado em Sociedade, Tecnologia e Meio Ambiente) - Centro Universitário de Anápolis, UniEvangélica, Anápolis.

ELIAKIM-IKECHUKWU, C.F., et al. The effect of aqueous ethanolic extract of Stachytarpheta cayennensis on the histology of the liver and fasting blood sugar of nondiabetic and diabetic wistar rats. The Internet Journal of Nutrition and Wellness. v. 10, 2010.

ELISABETSKY, E. Etnofarmacologia. Ciência e Cultura. v. 55, p. 35-36, 2003.

GOMES, K.O. et al. Atenção Primária à Saúde - a "menina dos olhos" do SUS: sobre as representações sociais dos protagonistas do Sistema Único de Saúde. Ciência \&
Saúde Coletiva. n. 16, p. 881-892, 2011.

GOULART, S. et al. Anti-inflammatory evaluation of Solidago chilensis Meyen in a murine model of pleurisy. Journal of Ethnopharmacology. v. 113, p. 346-353, 2007.

HENNEBELLE, T. et al. Antioxidant and Neurosedative Properties of Polyphenols and Iridoids from Lippia alba. Phytother. Res. v. 22, p.256-258, 2008.

HETLAND, G. et al. Protective Effect of Plantago major L. Pectin Polysaccharide against Systemic Streptococcus pneumoniae Infection in Mice. Scandinavian Journal of Immunology. v. 52, p. 348-355, 2000.

IGNÁCIO, S.R.N. et al. Nitric oxide production by murine peritoneal acrophages in vitro and in vivo treated with Phyllanthus tenellus extracts. Journal of Ethnopharmacology. v. 74, p. 181-187, 2001.

ISLAM, M. A. et al. Analgesic and anti-inflammatory activity of Leonurus sibiricus. Fitoterapia. v. 76, p. 359-362, 2005.

KAMEL, E.G. et al. Parasitological and biochemical parameters in Schistosoma mansoni-infected mice treated with methanol extract from the plants Chenopodium ambrosioides, Conyza dioscorides and Sesbania sesban. Parasitology International. v. 60, p. 388-392, 2011.

LIMA, C. B. et al. Uso de Plantas Medicinais pela População da Zona Urbana de Bandeirantes-PR. Revista Brasileira de Biociências. v. 5, supl. 1, p. 600-602, 2007.

LIZ, R. et al. The anti-inflammatory modulatory role of Solidago chilensis Meyen in the murine model of the air pouch. Journal of Pharmacy and Pharmacology. v. 80, p. 515-521, 2008.

MACHADO, D.G. et al. Antidepressant-like effect of the extract of Rosmarinus officinalis in mice: Involvement of the monoaminergic system. Progress in NeuroPsychopharmacology \& Biological Psychiatry. v. 33, p. 642-650, 2009.

MAIOLI, M.A. et al. Iron chelating-mediated antioxidant activity of Plectranthus barbatus extract on mitochondria. Food Chemistry. v. 122, p. 203-208, 2010.

OLIVEIRA H. B. et al. Ethnopharmacological study of medicinal plants used in Rosário da Limeira, Minas Gerais, Brazil. Brazilian Journal of Pharmacognosy. v. 20, n. 2, 2010.

QUINTANS JÚNIOR, L.J. et al. Antinociceptive and antiinflammatory effects of Costus spicatus in experimental animals. Pharmaceutical Biology. v.48, n.10, p.10971102, 2010.

RAGHAV, S.K. et al. Anti-inflammatory effect of Ruta graveolens L. in murine macrophage cells Journal of Ethnopharmacology. v. 104, n. 1, p. 234-239, 2006.

RINGBOM, T. et al. Ursolic Acid from Plantago major, a Selective Inhibitor of Cyclooxygenase-2 Catalyzed Prostaglandin Biosynthesis. Journal of Natural Products v.61, p.1212-1215, 1998.

ROERSCH, C.M.F.B. Piper umbellatum L.: A comparative cross-cultural analysis of its medicinal uses and an ethnopharmacological evaluation. Journal of Ethnopharmacology. v.131, p.522-537, 2010.

ROSA, C. et al. Representações e intenção de uso da Fitoterapia na atenção básica à saúde. Ciência \& Saúde Coletiva. v.16, n.1, p. 311-318, 2011. 
SAMUELSEN, A.B. The traditional uses, chemical constituents and biological activities of Plantago major L. A review. Journal of Ethnopharmacology. v. 71, p. 1-21, 2000.

SASAKI, K. et al. Rosmarinus officinalis polyphenols produce anti-depressant like effect through monoaminergic and cholinergic functions modulation. Behavioural Brain Research. v. 238, p. 86- 94, 2013.

SILVA, A.G. et al. Evaluation of an extract of Brazilian arnica ( Solidago chilensis Meyen, Asteraceae) in treating lumbago. Phytotherapy research. v. 24, n. 2, p. 283-287, 2010.

SILVA, B.P.; PARENTE, J.P. Bioactive polysaccharides from Costus spicatus. Carbohydrate Polymers. v. 51, p. 239-242, 2003.

SILVA, A. A. M. Apostila EPI INFOтм. Programa de PósGraduação em Saúde Coletiva - UFMA 3. Disponível em: <http://www.pgsc.ufma.br/arquivos/apostilaEpilnfo. pdf>. Acesso em: $21 \mathrm{dez} 2013$.

SOUSA, I.M.C. et al. Práticas integrativas e complementares: oferta e produção de atendimentos no SUS e em municípios selecionados. Caderno Saúde Pública. v. 28, p. 2143-2154, 2012.

TROTTER, R.; LOGAN, M. Informant consensus: a new approach for identifying potentially effective medicinal plants. In: ETKIN N. L. Indigenous medicine and diet: biobehavioural approaches. Nova York: Redgrave. p.91-111, 1986.
TÜREL, I. et al. Hepatoprotective and anti-inflammatory activities of Plantago major L. Indian Journal of Pharmacology. v.41, n.3, p.120-124, 2009.

VALEZE, F.H. \& BRENZAN, M.A. Perfil de utilização de medicamentos fitoterápicos pela população do município de Boa Esperança - PR. SaBios-Revista de Saúde e Biologia. v. 6, n.1, p.17-24, 2011.

VELASCO-LEZAMA, R. et al. Effect of Plantago major on cell proliferation in vitro. Journal of Ethnopharmacology. v. 103, p. 36-42, 2006.

VITALINI, S.; IRITI, M. et al. Traditional knowledge on medicinal and food plants used in Val San Giacomo (Sondrio,Italy) - An alpine ethnobotanical study. Journal of Ethnopharmacology. v. 145, p. 517-529, 2013.

WHO World Health Organization - CID 101996. Classificação estatística internacional de doenças e problemas relacionados à saúde. Disponível em: http://www.datasus.gov.br/cid10/V2008/cid10.htm. Acesso em: 06 abr 2013.

YUAN,L.P. et al. Protective effects of total flavonoids of Bidens pilosa L. (TFB) on animal liver injury and liver fibrosis. Journal of Ethnopharmacology. v.116, p. 539-546, 2008.

ZÉTOLA, M. et al. CNS activities of liquid and spray-dried extracts from Lippia alba-Verbenaceae (Brazilian false melissa). Journal of Ethnopharmacology. v.82, n.2, p.207-215, 2002.

Rev. Bras. PI. Med., Campinas, v.17, n.4, supl. II, p.928-936, 2015. 